\title{
Cytotoxic Activities of New Jadomycin Derivatives
}

\author{
Jian-Ting Zheng, Uwe Rix, Lixia Zhao, Cynthia Mattingly, Val Adams, Quan Chen, \\ Jürgen Rohr, Ke-Qian Yang
}

Received: April 3, 2005 / Accepted: May 24, 2005

(C) Japan Antibiotics Research Association

\begin{abstract}
Cytotoxic activities of jadomycin B and five new jadomycin derivatives against four cancer cell lines (HepG2, IM-9, IM-9/Bcl-2 and H460) were evaluated. Jadomycin $\mathrm{S}$ was most potent against HepG2, IM-9 and IM-9/Bcl-2 while jadomycin $\mathrm{F}$ was most potent against H460. Their potencies correlated with the degrees of apoptosis induced. Structure-activity-relationship analyses clearly demonstrate that the side chains of the oxazolone ring derived from the incorporated amino acids make a significant impact on biological activity. Therefore, jadomycin offers an ideal scaffold to manipulate structure and could be exploited to make many novel bioactive compounds with altered activities.
\end{abstract}

Keywords jadomycin, derivative, Streptomyces venezuelae, cytotoxic

Polyketides represent one of the largest families of secondary metabolites found in nature, and often possess interesting biological activities [1,2]. The angucycline group of polyketides, typically consist of a tetracyclic aglycone assembled in an angular manner, display a multitude of activities that include antibacterial, antitumor, antiviral and enzyme inhibitory activities [3, 4]. Jadomycin B (1, Fig. 1) is an angucycline-derived antibiotic produced by $S$. venezulae ISP5230 when grown in an isoleucinecontaining medium under stress conditions [5 7]. During its biosynthesis, an angucyclinone intermediate undergoes oxidative cleavage at ring $\mathrm{B}$ and an L-isoleucine is incorporated to generate the unique pentacyclic core of the jadomycin aglycon $[8,9]$. By substituting L-isoleucine in the jadomycin $\mathrm{B}$ production medium with other amino acids, eleven new jadomycin derivatives were generated [10]. Five of them (Fig. 1), designated jadomycins Ala (2), F (3), V (4), S (5) and T (6), which has incorporated alanine, phenylalanine, valine, serine and threonine, respectively, were characterized in detail [10]. The diastereomeric mixture with $3 \mathrm{a}-S$ and $3 \mathrm{a}-\mathrm{R}$ configuration found for these jadomycins derivatives along with the observed dynamic equilibrium (found for compound $\mathbf{1 , 2}$, 3, and 4) and the fact that various amino acids with different side chains can be incorporated into the jadomycin skeleton strongly support our hypothesis that the incorporation of amino acid most likely occurs nonenzymatically [10]. This exemplifies the potential to use jadomycin scaffold to generate novel structure modifications on the oxazolone ring. Although the numbers of angucycline compounds reported have grown to more
K.-Q. Yang (Corresponding author), J.-T. Zheng: State Key Laboratory of Microbial Resources, Institute of Microbiology, Chinese Academy of Sciences, Beijing, 100080, P.R. China and Graduate School of the Chinese Academy of Sciences, E-mail: yangkq@im.ac.cn

J. Rohr (Corresponding author), U. Rix: Department of Pharmaceutical Sciences, College of Pharmacy, University of Kentucky, 725 Rose Street, Lexington, Kentucky 40536-0082, U.S.A., E-mail: jrohr2@email.uky.edu
Q. Chen (Corresponding author), L. Zhao: Institute of Zoology, Chinese Academy of Sciences, Beijing 100080, P.R. China, E-mail: chenq@ioz.ac.cn

C. Mattingly, V Adamsd: Department of Pharmacy Practice and Science, College of Pharmacy, University of Kentucky, 725 Rose Street, Lexington, Kentucky 40536-0082, U.S.A. 


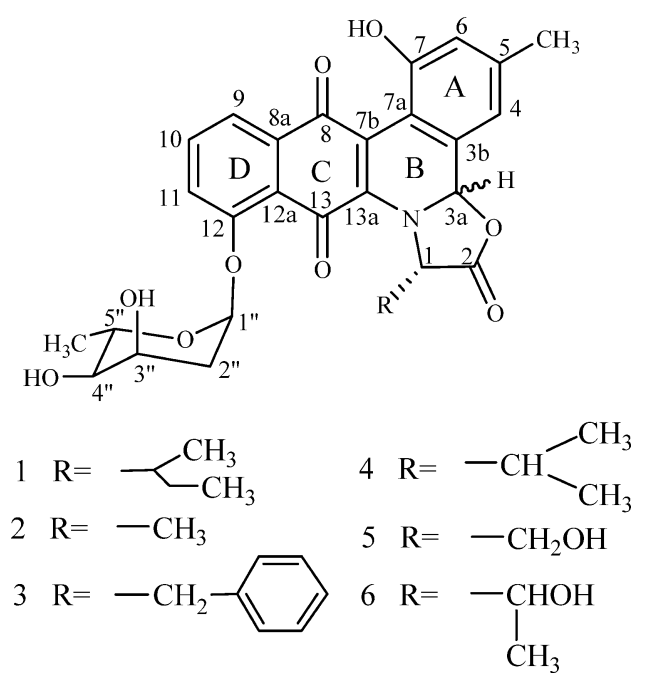

Fig. 1 Structures of jadomycin derivatives.

than 250 structurally defined representatives [3, 4], many of which showed antitumor activities in vitro, their modes of action are poorly understood. Even for the related anthracyclines, a group of well-characterized polyketides in term of biosynthesis and anti-tumor efficacy, the mechanisms underlying their activity are still far from clear [11]. Here, we report cytotoxic and pro-apoptotic activities of these jadomycin derivatives, thus providing interesting insights into the structure-activity-relationships of jadomycins.

As we have reported [10], by directed biosynthesis eleven new jadomycins were produced. Five of them including compounds $2,3,4,5$, and 6 were characterized by NMR. Other six analogs were only characterized by MS due to low yield and instability. The two diastereomer of compounds $1,2,3$, and 4 is inseparable while that of compounds $\mathbf{5}$ and $\mathbf{6}$ is chromatographically separable and $3 \mathrm{a}-R$ form constitutes more than $95 \%$ of them. So, compounds $1,2,3$, and 4 and $3 \mathrm{a}-R$ form of compounds 5 and $\mathbf{6}$ were selected to evaluate their biological activities. Previously, jadomycin B and A were reported to possess antibacterial and anti-yeast activities, we are also interested in their antitumor activities, and in their structure-activityrelationships. Multiple myeloma, liver and lung cancers are three of the leading causes of cancer-related deaths and continue to show increasing incidence. So, four human tumor cell lines including HepG2 (human hepatocellular carcinoma) ATCC HB-8065, IM-9 (human lymphoblast, immunoglobulin-secreting cell line derived from a multiple myeloma) ATCC CCL159, and IM-9 subline IM-9/Bcl-2 [12], and H460 (non-small-cell lung cancer) obtained from NCI were used to evaluate the cytotoxicity of these
Table $1 \quad I C_{50}$ values of jadomycin derivatives ${ }^{a}$

\begin{tabular}{lrrrrrc}
\hline & \multicolumn{7}{c}{$\mathrm{IC}_{50}(\mu \mathrm{M})^{\mathrm{b}}$} \\
\cline { 2 - 7 } Cell lines & $\mathbf{1}$ & $\mathbf{2}$ & $\mathbf{3}$ & $\mathbf{4}$ & $\mathbf{5}$ & $\mathbf{6}$ \\
\hline HepG2 & 10.8 & 100 & 49 & 27 & 9.8 & 27 \\
IM9 & 8.5 & 40 & 29 & 8.2 & 6.3 & 9.1 \\
H460 & 21.8 & 30.7 & 12.4 & $\mathrm{ND}^{\mathrm{b}}$ & 19.2 & 19.6 \\
\hline
\end{tabular}

${ }^{a}$ Human cancer cell lines HepG2, IM9 and H460 were treated continuously with jadomycin derivatives for 24 hours. Cytotoxicity was determined by the MTT/MTS assay as described in the text.

${ }^{b} I_{50}$ was calculated from survival curves of at least three independent experiments. ND, not determined.

jadomycin derivatives. Currently, therapeutic management of these cancers is often impeded by multi-drug resistance, even without previous drug treatment. Bcl-2 overexpressing cells (IM-9/Bcl-2) were included because these cells were resistant to conventional chemotherapy [13]. Compounds active against Bcl-2 over-expressing cells are of special interest. The sensitivity of above cell lines to jadomycin derivatives was examined by continuous exposure to a range of drug concentrations, and monitoring cell survival after 24 hours by the 3-(4,5-dimethylthiazo-2yl)-2,5-diphenyltetrazolium bromide (MTT) or 3-(4,5dimethylthiazol- 2-yl)-5-(3-carboxymethoxyphenyl)-2-(4sulfophenyl)- $2 H$-tetrazolium (MTS) assay. The adherent cells were assessed by MTT assay and the floating cells by MTS assay. For MTT assay, exponentially growing cells $\left(1 \times 10^{4} \sim 2 \times 10^{4}\right.$ cells $\left./ 100 \mu \mathrm{l}\right)$ were seeded in 96-well plates and incubated for 12 hours. Cells were then treated continuously with jadomycin derivatives. After 24 hours, $10 \mu 124 \mathrm{mM}$ MTT in $0.9 \%$ sodium chloride was added to the medium. After 2 to 4 hours of incubation at $37^{\circ} \mathrm{C}$, the culture medium and MTT were removed and $100 \mu 1 \mathrm{DMSO}$ was added to the wells to dissolve the precipitate of reduced MTT, then the absorbance of DMSO solution was determined at $570 \mathrm{~nm}$ with a microplate reader (Wellscan k3, KHB). The MTS assay followed procedures from Promega with the CellTiter 96 AQueous One Solution Cell Proliferation Assay and absorbance was determined at 492 $\mathrm{nm} . \mathrm{IC}_{50}$ was calculated as the concentration of drug causing a $50 \%$ inhibition in absorbance compared to the untreated control. Table 1 summarizes the average concentrations of jadomycin derivatives required to inhibit $50 \%$ of cell growth, pooled from independent experiments. Among the analogues tested, $\mathrm{IC}_{50}$ values varied from $6.3 \mu \mathrm{M}$ to $100 \mu \mathrm{M}$. Compound $\mathbf{3}$ was most potent against $\mathrm{H} 460\left(\mathrm{IC}_{50} 12.4 \mu \mathrm{M}\right)$, whereas compound 5 was most 
active against $\mathrm{IM}-9\left(\mathrm{IC}_{50} 6.3 \mu \mathrm{M}\right)$ and $\mathrm{HepG} 2\left(\mathrm{IC}_{50}\right.$ $9.8 \mu \mathrm{M})$. Compound $\mathbf{1}$ was second to $\mathbf{5}$ in potency against HepG2 and IM-9. Compound $2\left(\mathrm{IC}_{50} 30.7 \mu \mathrm{M}\right.$ against $\mathrm{H} 460 ; 100 \mu \mathrm{M}$ against HepG2 and $40 \mu \mathrm{M}$ against IM-9) was the least active toward all three cell lines. It is interesting that all the compounds showed activity against IM-9/Bcl-2 cells (relatively rare among known compounds), although the $\mathrm{IC}_{50}$ s were not impressive (more than $100 \mu \mathrm{M}$ ). Figure 2 shows the survival curves of IM-9/Bcl-2 cells treated with these compounds.

Apoptosis is a cell-suicide mechanism which plays a critical role in normal development, tissue homeostasis and antitumor therapeutics [14]. Deregulation of this process

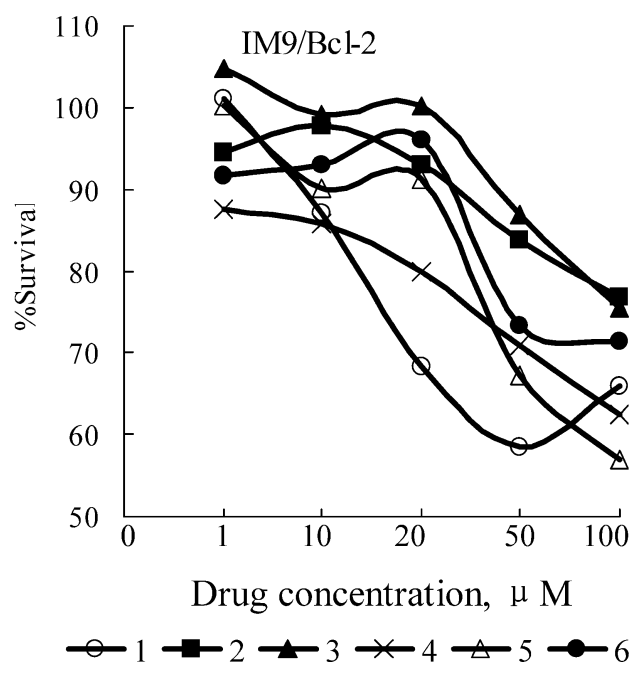

Fig. 2. Dose-response antiproliferative activities of jadomycin derivatives. $1 \mathrm{M} 9 / \mathrm{Bcl}-2$ cells were treated continuously with jadomycin derivatives at various concentrations. Twenty-four hours later, cytotoxicity was determined by the MTT/MTS assay as described in the text. results in cancer, neurodegenerative diseases or other pathological conditions. Induction of tumor cell apoptosis by anti-tumor drugs is the typical effect of antitumor therapeutics. In order to ascertain whether the cytotoxic potency of jadomycin derivatives was mediated by induction of apoptosis, HepG2, IM-9 and IM-9/Bcl-2 cells were treated with these compounds and the percentage of apoptotic cells was determined by fluorescein isothiocyanate (FITC)-Annexin V/propidium (PI)-double staining methods [13]. Flow cytometric analysis was performed by monitoring the green fluorescence of FITC conjugated Annexin V $(530 \pm 30 \mathrm{~nm})$ and the red fluorescence of DNA bound PI $(630 \pm 22 \mathrm{~nm})$. Live cells (unstained with either fluorochrome) were considered discriminated from apoptotic cells (stained only with annexin-V) and necrotic cells (stained with both Annexin$\mathrm{V}$ and PI). Staining of cells by PI was an indicator of the loss of plasma membrane integrity. All data were analyzed with Cell Quest software (BD). The results (Table 2) show the abilities of these compounds to induce apoptosis correlated with their cytotoxic activities. Compound $\mathbf{1}$ and 5 were much more active than others in inducing apoptosis, while compound $\mathbf{2}$ was the least active. It is noteworthy, $\mathbf{5}$ was most active against IM-9/Bcl-2, and could induce obvious apoptosis of IM-9/Bcl-2; while 1 was more active against IM-9 and HepG2.

The need to develop new therapeutically active agents provides impetus to enlarge the reservoir of novel bioactive secondary metabolites. All the jadomycin derivatives tested here could suppress proliferation of tumor cells in vitro. Compound 2, which has a simple methyl group at the oxazolone ring, is the least active among these compounds. A hydroxyl substitution of a methyl hydrogen in compound $\mathbf{2}$, resulting in compound $\mathbf{5}$ (serine derivative) improved the activity against HepG2 cells nearly ten fold, highlighting

Table 2 Apoptosis of IM-9, IM-9/Bcl-2 and HepG2 cells treated with jadomycin derivatives ${ }^{\text {a }}$

\begin{tabular}{|c|c|c|c|c|c|c|c|c|c|c|c|c|c|c|}
\hline \multirow{3}{*}{ Cell lines } & \multicolumn{14}{|c|}{$\%$ Apoptotic cells } \\
\hline & \multicolumn{2}{|c|}{ Control } & \multicolumn{2}{|c|}{1} & \multicolumn{2}{|c|}{2} & \multicolumn{2}{|c|}{3} & \multicolumn{2}{|c|}{4} & \multicolumn{2}{|c|}{5} & \multicolumn{2}{|c|}{6} \\
\hline & $E A^{b}$ & $N L A^{c}$ & EA & NLA & EA & NLA & EA & NLA & EA & NLA & EA & NLA & EA & NLA \\
\hline IM-9 & 3.9 & 3.5 & 25.4 & 36.9 & 13.8 & 11.2 & 16.6 & 11.8 & 22.8 & 15.9 & 29.0 & 19.2 & 15.7 & 14.1 \\
\hline $\mid \mathrm{M}-9 / \mathrm{Bc|}-2$ & 2.0 & 2.8 & 7.9 & 4.2 & 3.3 & 2.7 & 6.2 & 2.7 & 11.1 & 3.3 & 19.5 & 6.9 & 8.3 & 4.0 \\
\hline HepG2 & 2.5 & 5.0 & 19.1 & 12.9 & 4.4 & 6.7 & 5.9 & 9.1 & 7.2 & 9.2 & 16.2 & 9.7 & 4.8 & 7.9 \\
\hline
\end{tabular}

a IM-9 and HepG2 were treated with $10 \mu \mathrm{M}$ of jadomycin derivatives, IM-9/Bcl2 with $20 \mu \mathrm{M}$. The percentage of apoptotic cells was determined as average of at least three independent experiments.

${ }^{b}$ EA, cells in early stages of apoptosis, annexin- $\mathrm{V}^{+} / \mathrm{PI}^{-}$.

${ }^{\mathrm{c}} \mathrm{NLA}$, cells in necrosis/late apoptosis, annexin- $\mathrm{V}^{+} / \mathrm{PI}^{+}$ 
the importance of the hydroxyl residue in compound $\mathbf{5}$. The lower activity of compound $\mathbf{6}$ (similar to $\mathbf{5}$ in possessing a hydroxyl group in the side chain) maybe due to spatial hindrance caused by the additional methyl group of the threonine residue. The original compound $\mathbf{1}$, which has a $1-$ methylpropyl side chain at the oxazolone ring, is one of the most active compounds against IM-9 and HepG2, but its activity is clearly distinguished from that of $\mathbf{5}$ for being less effective against cells overexpressing Bcl-2. The phenylalanine-derived compound $\mathbf{3}$, the most active compound against H460, has an additional phenyl residue compared to the least active 2 . Since compounds $\mathbf{1}, \mathbf{3}$ or $\mathbf{5}$ were each more active against a different cell line, they could act on different targets or generate different effects upon binding to their targets in a specific background. Due to scarcity of knowledge about their target(s) and interactions, we cannot speculate as to the structureactivity-relationship at this point. We also tested the influence of these compounds on cell cycle and mitochondrion function (data not shown); no significant effect was observed indicating that these compounds did not directly impact the cell cycle or the mitochondria. However, it can be safely concluded that all jadomycins tested are cytotoxic against life threatening cancer cells (including Bcl-2 over expressing cells) and that the side chain on the oxazolone has a significant effect on that activity. Therefore, substitution of the amino acid derived side chain ( $\mathrm{R}$ in Fig. 1) on the oxazolone ring by directed biosynthesis is a feasible method to generate novel bioactive jadomycins.

Acknowledgments We wish to thank Professor Xiu-Fen Kou for her assistance with purification methods, Mr. Li Xiao-Ming for his assistance with LC-MS analyses and Mrs. Wang Jing for her assistance operating flow cytometry. This work was supported by start-up funding from Institute of Microbiology and Chinese Academy of Sciences to KQY, and by the NIH and the Kentucky Lung Cancer Research Foundation to JR.

\section{References}

1. Borchardt JK. Combinatorial biosynthesis panning for pharmaceutical gold. Mod Drug Discov 2: 22-29 (1999)

2. Hopwood DA, Sherman DH. Molecular genetics of polyketide and its comparison to fatty acid biosynthesis.
Annu Rev Genet 24: 37-66 (1990)

3. Krohn K, Rohr J. Angucyclines: total syntheses, new structures, and biosynthetic studies of an emerging new class of antibiotics. Topics Curr Chem 188: 127-195 (1997)

4. Rohr J, Thiericke R. Angucycline group antibiotics. Nat Prod Rep 9: 103-137 (1992)

5. Ayer SW, McInnes AG, Thibault P, Walter JA, Doull JL, Parnell T, Vining LC. Jadomycin, a novel $8 H$ benz[b]oxazolo[3,2-f]phenanthridine antibiotic from Streptomyces venezuelae ISP5230. Tetrahedron Lett 32: 6301-6304 (1991)

6. Doull JL, Ayer SW, Singh AK, Thibault P. Production of a novel polyketide antibiotic, jadomycin B, by Streptomyces venezuelae following heat shock. J Antibiot 46: 869-871 (1993)

7. Doull JL, Singh AK, Hoare M, Ayer SW. Conditions for the production of jadomycin B by Streptomyces venezuelae ISP5230: effects of heat shock, ethanol treatment and phage infection. J Ind Microbiol 13: 120-125 (1994)

8. Han L, Yang K, Ramalingam E, Mosher RH, Vining LC. Cloning and characterization of polyketide synthase genes for jadomycin B biosynthesis in Streptomyces venezuelae ISP5230. Microbiol 140: 3379-3389 (1994)

9. Yang K, Han L, Ayer SW, Vining LC. Accumulation of the angucycline antibiotic rabelomycin after disruption of an oxygenase gene in the jadomycin B biosynthetic gene cluster of Streptomyces venezuelae. Microbiol 142: 123-132 (1996)

10. Rix U, Zheng J, Remsing Rix LL, Greenwell L, Yang K, Rohr J. The dynamic structure of jadomycin B and the amino acid incorporation step of its biosynthesis. J Am Chem Soc 126: 4496-4497 (2004)

11. Gewirtz DA. A critical evaluation of the mechanisms of action proposed for the antitumor effects of the anthracycline antibiotics adriamycin and daunorubicin. Biochem Pharmacol 57: 727-741 (1999)

12. Chen Q, Gong B, Almasan A. Distinct stages of cytochrome $C$ release: evidence of the feedback of the amplification of cytochrome $\mathrm{C}$ release from mitochondria by caspases linking to the dysfunction of mitochondria. Cell Death Differ 7: 227-233 (2000)

13. Chen Q, Gong B, Zhou A, Hussein M, Almasan A. RAIL/Apo2L induction and modulation of Bcl-2-related proteins regulates interferon-induced apoptosis in multiple myeloma. Blood 98: 2183-2192 (2001)

14. Hipfner DR, Cohen SM. Connecting proliferation and apoptosis in development and disease. Nat Rev Mol Cell Biol 5: 805-815 (2004) 